\title{
THE ROLE OF COMPOUND NUCLEI IN INTERMEDIATE-ENERGY FEAVY-ION REACTIONS
}

\author{
Luciano G. Moretto and Gordon J. Wozniak \\ Nuclear Science Division, Lawrence Berkeley Laboratory, University of California, Berkeley, \\ California, 94720, USA
}

Hot compound nuclei are frequently produced in intermediate-energy reactions through a variety of processes. Their decay is shown to be an important and at times dominant source of complex fragments, high energy-gamma rays, and even pions.

\section{INTRODUCTION}

Intermediate-energy heavy-ion reactions have confronted us with a wealth of phenomena unmatched by the lower energy regime. The tidy picture of low-energy nuclear physics is framed by a few basic mechanisms. At one extreme we have direct reactions, involving a narrow subset of nuclear modes, typically single particle degrees of freedom. In between we have quasi-elastic and deep-inelastic reactions involving a much larger number of modes, both single particle and collective, and associated with a much more profound degree of relaxation. At the other extreme we have compound nucleus (CN) processes, in which there is full relaxation of all the modes, and which are characterized by a complete decoupling between entrance and exit channels.

At intermediate energies this simple picture seens 10 disappear, drowned by a florid overgrowth of exit channels. To the untrained eye, such complexity can create irresistible images of new and exotic processes. For example, the variety and abundance of complex fragments produced in these reactions suggested mechanisms like the shattering of glass-like nuclei, ${ }^{1}$ or the condensation of droplets out of a saturated nuclear vapor, ${ }^{2}$ or the somewhat equivalent picture of a nuclear soup curding simultaneously into many fragments. 3,4 Such images were, and perhaps still are, so powerful that they thrived on themselves rather than on experiment. The word "multifragmentation" became very popular despite the perplexing lack of evidence for truly multi-fragment exit channels. This exuberance, initially a welcome indicator of the vitality of our field, should be tempered by a more sober interpretation of the data.

Complexity is not synonymous with novelty! Caution should be used by assuring oneself that the complexity of the reactions under study is not due to the proliferation and overlapping of conventional processes made possible by the large available energy. More than ever, it is necessary to assess the "background" of conventional processes before a new theory is declared proven or a new mechanism prematurely discovered! In particular, one would be well advised to check how large is the CN contribution to the production of complex fragments, gamma rays and even pions.

- Supported by the Director, Office of Energy Research, Division of Nuclear Physics of the Office of High Energy and Nuclear Physics of the U. S. Department of Energy under Contract DE-AC03076SF00098. 
This is the main topic of this paper and we shall dwell on it at length.

At this juncture it may be useful to remark on a now widespread tendency to compare data directly with more or less "ab initio" calculations, like mean field theories with collision terms, etc. In our opinion this is at best not useful, and is at worst quite dangerous. If we had the "exact" theory describing heavy ion reactions on the basis of the nucleon-nucleon interaction, we would be able, by definition, to fit the data perfectly. In fact, a calculation on a computer would be equivalent to the corresponding experiment performed at an accelerator. However, if we do not understand the experiment, it is truly of little consequence to know that our calculation refroduces it correctly. What we would have proved is that the nucleon-nucleon interaction used in our calculation is adequate.

This underscores the need for empirical macroscopic categories which are solidly based upon experimental observations and which allow for a simple classification and quantitative assessment of the data over a broad range of experimental environments. The $\mathrm{CN}$ is one of such empirical categories that has proven its usefulness at low energies and, in our belief, is still an extremely useful concept at intermediate energies. An "ab initio" calculation could very well go through a "compound nucleus" stage unbelanownst to us and to itself. And yet the empirical verification of whether a $\mathrm{CN}$ is involved or not is most relevant to our understanding.

As a final remark, in the complex and confusing experimental environment characteristic of intermediate energies, it is profitable and often necessary to choose the reactions judiciously. A little ingenuity in such a choice may emphasize the process one intends to study and minimize the disturbing noise arising from "irelevant" features of the reactions. For instance efforts to limit the number of sources of complex fragments or to make their identification easier may be quite beneficial.

\section{COMPOUND NUCLEI AT INTERMEDIATE ENERGIES}

The degree of energy relaxation th:s can be achieved in nuclear reactions is extraordinary indeed! Even the rather commonplace $\mathrm{CN}$ produced by bombarding a medium mass nucleus with $80-100$ MeV alpha particles is in a way already surprising, but the amount of energy deposited into internal degrees of freedom by heavy-ion reactions is, at times staggering. In the reaction $100 \mathrm{Mo}+100 \mathrm{Mo}$ at 23.4 A MeV as much as $800 \mathrm{MeV}$ or $\sim 4 \mathrm{MeV} /$ nucleon is deposited as excitation energy. 5,6 The use of neutron multiplicity detectors has allowed one to determine with a fair degree of accuracy the extent of energy thermalization. ${ }^{7}$ The conclusion, from this and similar charged particle measurements is that at intermediate energies, the energy relaxation is pervasive and profound. This, by itself does not mean that a $\mathrm{CN}$ hás been formed, since energy relaxation is only a necessary but not sufficient condition for its formation.

In the same way, the presence of evaporation-like particle spectra or a fission-like binary decay are not by themselves sufficient critieria. The presence of a $\mathrm{CN}$ can be tested by verifying the statistical competition of all the decay channels, or at least the statistical competition of a rather improbable channel (like the emission of a moderate mass complex fragment or the emission of an energetic gamma ray or pion) against a dominant channel like neutron or proton emission. Because 
of these considerations, the determination of absolute cross sections or, even better, of excitation functions is essential.

How can CN be formed at intermediate energies?

At low energies we are used to preparing $\mathrm{CN}$ by means of fusion reactions; after all, it is not an accident that $\mathrm{CN}$ are called compound. However, what Bohr had in mind when he introduced this new concept was not the particular way in which the CN was formed, as through fusion. To the contrary he stressed that, due to the complete equilibration of the system, all the dynamical information associated with the entrance channel was forgotien, and that the decay could only depend upon the statistical features of the available exit channels. In order to prove that it does not matter how she $\mathrm{CN}$ is formed, the early and not so early literature is rich with examples of different "fusion" channels leading to the same $\mathrm{CN}$ - which does indeed decay always in the same way. So, the essence of the compound nucleus is not in the fusion of target and projectile but in the decoupling of the Entrance and Exit Channels.

Having accepted this, we realize that $\mathrm{CN}$ may be more common than previously thought. For instance:

1) The residue product after a $\mathrm{CN}$ evaporates a particle is still a $\mathrm{CN}$.

2) The two fragments produced in fission relax and eventually evaporate neutrons as $\mathrm{CN}$.

3) Quasi-elastic and deep-inelastic heary ion reactions produce fragments which also relax into $\mathrm{CN}$ and decay as such.

4) In the process of incomplete fusion both the incomplete fusion product and the spectator do eventually relax into $\mathrm{CN}$.

5) In the fireball production mechanism, the two spectator fragments are expected to relax into $\mathrm{CN}$, and even the fireball may not be far from a $\mathrm{CN}$, either.

In view of the above, we are going to explore at intermediate energies the role of $\mathrm{CN}$ emission in a) complex fragment production; b) garna-ray emisson and c) pion emission.

\section{COMPLEX FRAGMENT PRODUCTION}

With the advent of intermediate energies, complex fragments have become a very pervasive presence. Where could they possibly come from? Not from $\mathrm{CN}$, since conventional wisdom held that $\mathrm{CN}$ decay solely by $\mathrm{n}, \mathrm{p}$, and alpha-particle emission or by fission. As a consequence, complex fragments could only come from some other novel mechanism, like liquid-vapor equilibrium, multifragmentation, ete. ${ }^{8}$ However, at low energy it has been shown that $\mathrm{CN}$ can emit complex fragments. ${ }^{9}$ In fact, it is possible to consider light fragment emission and fission as the two extremes of a single mode of decay, connected by the mass asymmetry degree of freedom. ${ }^{10}$ This process allows for complex fragment emission and the ranity of its occurence is due to the important but accidental fact of the high barriers associated with such an emissions.

Let us consider the potential-energy surface of a nucleus as a function of a suitable set of deformation coordinates. This surface is characterized by the ground state mininium and by the fission saddle point. We can cut this surface with a line passing through the fission saddle point along the mass-asymmetry coordinate in such a way that each of its points is a saddle point if one 
freezes the mass-asymmetry coordinate. The locus of all these conditional saddle points we call the "ridge line". ${ }^{10}$ Fig. 1 shows two examples of this line (solid curves), one for a light system below the Businaro-Gallone point and the other for a heavier system above the Businaro-Gallone point. The same figure shows the expected particle yield (dashed curves) following the statistical prediction: $\quad Y(Z) \propto \exp [-\mathrm{V}(\mathrm{Z}) / \mathrm{T}]$.

One can make three observations:

1) Systems below the Businaro-Gallone point give rise to a U-shaped mass or charge distribution with a minimum at symmetry.

2) Systems above the Businaro-Gallone point give rise to a similar distriburion but with a maximum (fission peak) growing in at symunetry.

3) The yield increases with temperature and increases fastest for the highest barriers.

Consequently complex fragments, although very rare at low energy, become rapidly abundant at high energies. The existence of this CN mechanism at low energies has been proven in detail. ${ }^{9}$ Could the fragments observed at higher energies arise from the same mechanism?

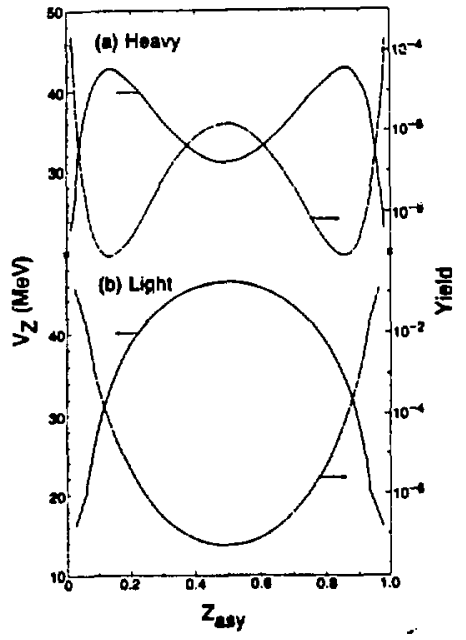

FIGURE 1

Schematic ridge line potentials (solid curve) and calculated yields (dashed curve) for: a) a heavy $\mathrm{CN}$ above the Businaro-Gallone point; and b) a light CN below the Businaro-Gallone point as a function of the mass-asymmetry coordinate $\left(\mathrm{Z}_{\text {asy }}\right)$.

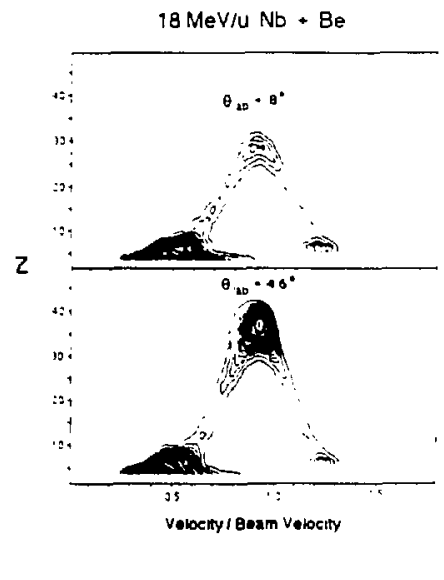

FIGURE 2

Contours of the invariant cross section in the $Z$ - velocity plane for complex fragments emitted from the $18 \mathrm{MeV} / \mathrm{u}{ }^{93} \mathrm{Nb}+{ }^{9} \mathrm{Be}$ reaction at $\theta_{1 \mathrm{ab}}$ $=4.6^{\circ}$ and $8^{\circ}$. The "big foot" visible at low velocities for $Z<10$ is attributed to quasi-elastic and deep-inelastic products. 
In experiments up to $50 \mathrm{~A} \mathrm{MeV}, 11$ we have been able to identify three kinds of sources of complex fragments, which turn out to be rather conventional. The three sources are:

1) Quasi-elastic/deep-inelastic scattering.

2) Spectators in incomplete-fusion processes.

3) Hot compound nuclei.

The first two sources produce fragments which are target and/or projectile related. The thind is just the high energy version of the low energy $\mathrm{CN}$ decay. How can these three sources be distinguished? We have found that reverse kinematies and very asymmetric target-projectile combinations are particularly useful for a series of reasons. The principal reasons are: 1) the quasi-elastic/deep-inelastic processes are confined to both low and high $Z$-values, whereas the incomplete-fusion spectators are confined to low Z-values leaving uncontaminated the intermediate Z-range for CN products; 2) The associated limited range of impact parameters leads to a corresponding narrow range of momentum transfers and consequently to a small range of source velocities; 3) keverse kinematics brings all the fragments inco a relatively narrow forwand cone and boosts their energy, thus greatly simplifying their detection and identification.

The evidence of the CN origin of these fragments can be seen in Fig. 2, where the cross section in the $\mathbf{Z}$ - velocity plane is shown for the reaction $18 \mathrm{~A} \mathrm{MeV}{ }^{93} \mathrm{Nb}+{ }^{9} \mathrm{Be}$ at two different angles. The two legs of the lambda partem represent the upper and lower solutions in reverse kinematics associated with the binary decay of the source, and correspond to the Coulomb circles visible in the $v_{11}-v_{1}$ plane for each $Z$ value in Fig. 3 for the $18 \mathrm{~A} \mathrm{MeV}{ }^{139} \mathrm{La}$ $+{ }^{12} \mathrm{C}$ reaction. The telltale signature of a binary decay is not only the presence of a sharp Coulomb circle, but the fact that its radius decreases with increasing $Z$ value as required by momentum conservation. The large cross sections observed at low $Z$ values and attached

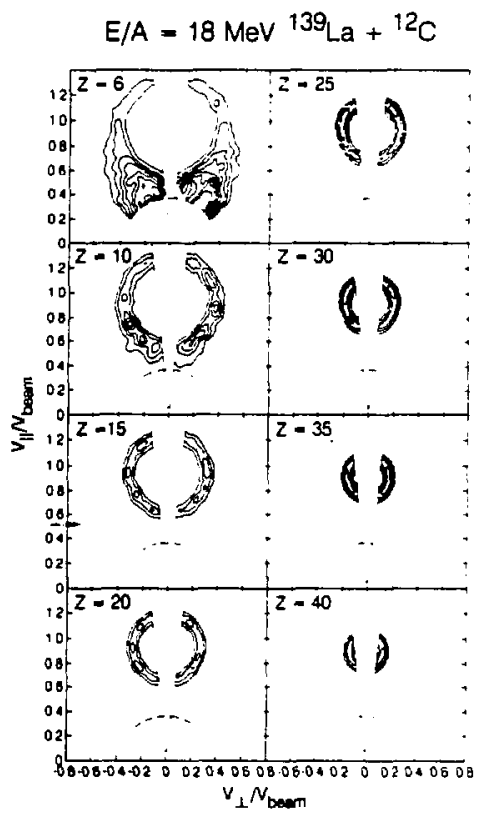

\section{FIGURE 3}

Contours of the experimental cross section $\partial^{2} \sigma / \partial V_{11} \partial V_{1}$ in the $V_{11}-V_{\perp}$ plane for representative fragment $Z$-yalues detected in the reaction $18.0 \mathrm{~A} \mathrm{MeV}{ }^{139} \mathrm{La}+{ }^{12} \mathrm{C}$. The beam direction is vertical. The dashed lines show the maximum and minimum angular thresholds and the low velocity threshold of the detectors. The magnitudes of the contour levels indicated are relative. 
to the low velocity branch (see Fig. 2) are associated with quasi-elastic and deep-inelastic products. The choice of very asymmetric target-projectile combinations shows here its wisdom. The more symmetric the target-projectile combination is, the more extensive the obscuration of the $\mathrm{CN}$ component by quasi-elastic \& deep-inelastic fragments is expected to be.

The centers of the circles give the source velocities which are remarkably independent of the fragment $\mathbf{Z}$ value $^{\mathbf{8}, 11}$ and correspond to either complete or incomplete fusion of the light target with the heavy projectile. The nearly linear dependence of the radii of the circles on the fragment $Z$-value demonstrates their Coulomb origin. ${ }^{8,11}$

The cross sections and their dependence upon energy and fragment Z-value are of particular importance to demonstrate their $\mathrm{CN}$ origin. When a $\mathrm{CN}$ is about to decay, it is offered many channels which will be chosen proportionally to their associated phase space. In particular, neutron, proton, and alpha-particle decay, because of their small associated barriers, are the dominant decay channels with which complex fragments must compete. Thus, the cross section associated with the emission of any given fragment reflects this competition. In Figs. $4 a$ \& 4 b an example of the absolute charge distributions are given, together with calculations performed with the $\mathrm{CN}$ decay code

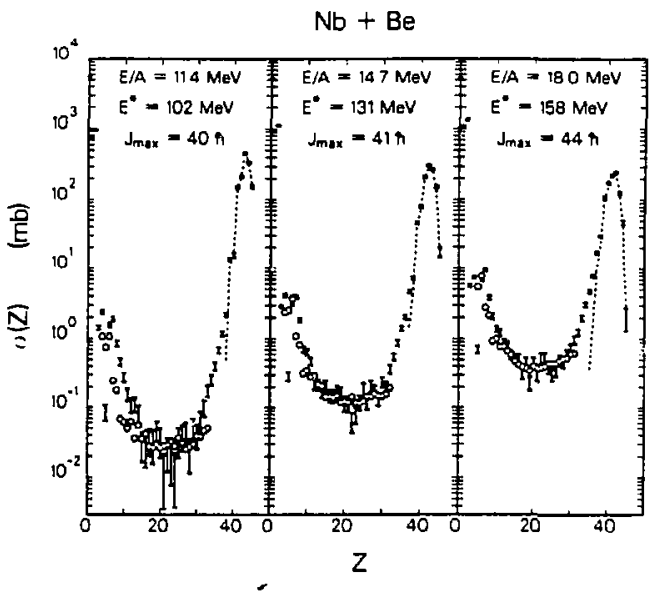

FIGURE 4a

Comparison of experimental and calculated charge distributions for the ${ }^{93} \mathrm{Nb}+{ }^{9} \mathrm{Be}$ reaction at E/A $=11.4,14.7$, and 18.0. The experimental data are indicated by the hollow circles and the values calculated with the code GEMINI are shown by the error bars. The dashed curve indicates the cross sections associated with classical evaporation residues which decay only by the emission of light particles $(Z \leq 2)$. Note the value of the excitation energy $\left(E^{*}\right)$ corresponding to complate fusion and the value of $J_{\max }$ assumed to fit the data. 
(GEMINI) ${ }^{11}$ which follows the decay of the CN through all the charnels including complex fragment emission. The code accurately reproduces the shape, magnitude, charge and energy dependence of the absolute cross sections, thus confirming $\mathrm{CN}$ decay as the dominant mechanism in this energy range.

Coincidence data confirm the binary nature of the decay. The $Z_{1}-Z_{2}$ scatter plots (see Fig. 5) show the diagonal band characteristic of binary decay. The hatched area is the predicted locus of events after correcting for sequential evaporation from the primary fragments. The spectrum associated with the sum $Z_{1}+Z_{2}$ shows a rather sharp peak very near the value of $Z_{\text {total }}$ indicating that there is only a small charge loss and that most of the total charge available in the entrance charnel is found in the two exit-channel partners.

The evidence presented above is but a small sample of the evidence available for $\mathrm{CN}$ emission of complex fragments at bombarding energies up to $50 \mathrm{~A} \mathrm{MeV}{ }^{8.11}$ So far binary decay has dominated the picture while multifragmentation has been conspicuously absent. Yet it is not unreasonable to envision, at even higher energies, exit channels with more than two main fragments. Does that mean, automatically, that the role of the $\mathrm{CN}$ is over? Most likely not!

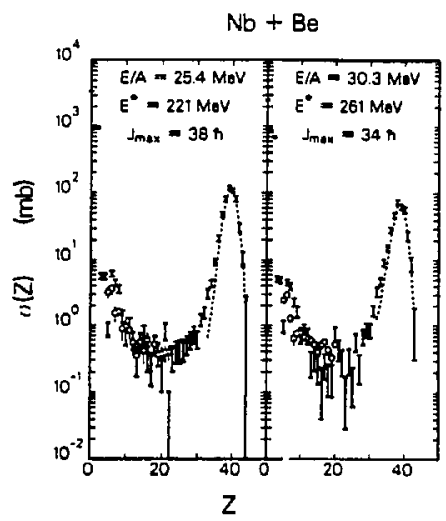

FIGURE 4b

Same as Fig. 4a except for $E / A=25.4$ and 30.3 .

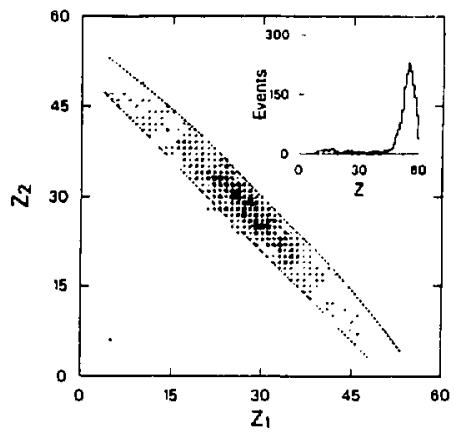

FIGURE 5

Scatter plots of the coincidence events, $Z_{1}-Z_{2}$, detected in two telescopes on opposite sides of the beam, for the ${ }^{139} \mathrm{La}+{ }^{12} \mathrm{C}$ reaction at $50 \mathrm{~A}$ $\mathrm{MeV}$. The hatched area is the predicted locus of events after correcting for sequential evaporation from the primary fragments. The distribution of the sum of the charges $\left(Z_{1}+Z_{2}\right)$ is shown in the inset. 


\section{MULTIFRAGMENTATION AND \\ NUCLEAR COMMINUTION}

The evidence presented so far illustrates the emission of complex fragments through binary CN decay. If there is enough excitation energy available, the primary binary-decay products are also very excited and have a significant probability of decaying in tum into two fragments. In this very conventional way, one can foresee one possible explanation for several fragments in the exit channel (multifragmentation), namely several sequential binary decays. At high energies, we expect a substantial yield for this mode, which could be responsible for a substantial background to other predicted multifragmentation mechanisms.

This process of sequential binary decay, controlled at each stage by the $\mathrm{CN}$ branching ratios, we call "nuclear comminution".8 The calculations of the resulting mass distributions are trivial although tedious and time consuming. We have tried to simulate the process by assuming a potential energy curve vs mass asymmetry (ridge line) with a maximum value of $40 \mathrm{MeV}$ for symmetry and 8 MeV for the extreme asymmetries. The primary yield curve is taken to be of the form:

$$
Y(A)=K \exp [-V(A) T(A)] \text {. }
$$

Each of the resulting fragments is assumed to have a similar ridge line, a properly scaled temperature, and is allowed to decay accordingly, until all the excitation energy is exhausted. For a series of initial excifation energies, the resulting mass distributions are shown in Fig. 6a. The log-log plots show an exquisite power-law dependence for the low mass fragments. At excitation energies of about $400 \mathrm{MeV}$, the exponents (see Fig. 6b) are around 2.3 - 2.4 which, incidentally, are

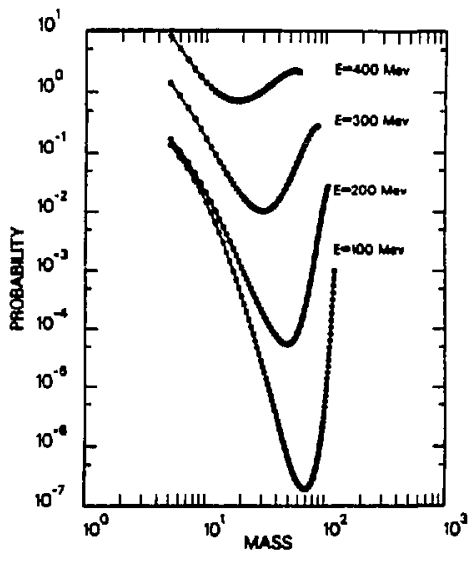

FIGURE 6a

Theoretical mass distributions from comminution calculations of the dexcitation of a CN with mass 150 at several excitation energies. Notice the power-law behavior at small masses.

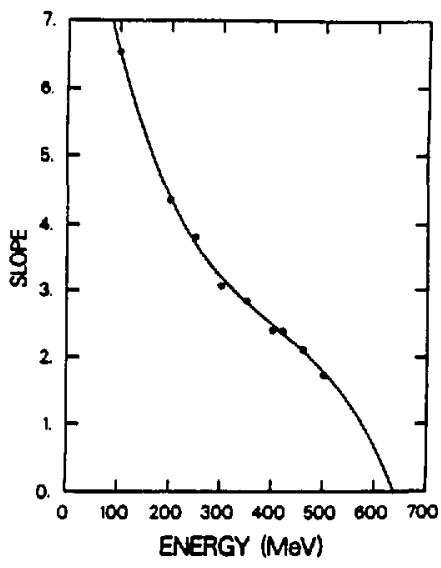

FIGURE $6 b$

Exponent $\tau$ of the power-law dependence as a function of excitation energy. 
very close to the value expected for the liquid-vapor phase transition at the critical temperature. This result shows that a power-law dependence is not a unique diagnustic feature of liquid-vapor equilibrium, but rather is an apparently "generic" property arising even from sequential binay decay or comminution. A more realistic calculation with the statistical code GEMINI leads to similar resuits. $^{8}$ With this code it is possible to calculate the excitation energy depentence of the binary, ternary, quaternary decays, etc. These excitation functions should be helpful in verifying the mechanism of nuclear comminution in the experimental data.

\section{HIGH-ENERGY $\%$ RAY EMISSION}

High energy gamma rays associated with intermediate-energy heavy-ion reactions were studied initially in order to observe the theoretically predicted "coherent bremsstrahlung" 12 associated with the collective deceleration of the two partners in the collision. Nature's lack of cooperation forced the interpretation of the data back to the less exalted "incoherent nucleon-nucleon bremsstrahlung" 12 which had at least the glamour of being associated with the entrance channel. This interpretation is probably correct in many cases. However, in reviewing the data available in the literature, we were struck by the possibility that some of the high energy gamma rays could come from the excited CN present in the exit channel. Unfortunately in all of these experiments the exit channels were too poorly characterized to permit any serious analysis of this sort.

Eventually we found an experiment, ${ }^{92} \mathrm{Mo}+{ }^{9} \mathrm{Mo}$ at $19.5 \mathrm{~A} \mathrm{MeV}{ }^{13}$, where the exit channel was well characterized. In this reaction the two nuclei undergo a deep-ineiastic collision. The dissipated energy which may amount to as much as $800 \mathrm{MeV}$ ( $400 \mathrm{MeV} / \mathrm{fragment!)}$ is disposed of mainly by sequential light particle emission. This emission is a true evaporation from the two deep inelastic fragments and has been studied in detail as a function of exit channel kinetic energy. ${ }^{5}$ At times these excited fragments emit complex fragments giving rise to a 3-body and a 4-body exit channel. ${ }^{6}$ This emission is also statistical and is in competition with the main decay channels like $n, p$, and alpha-particle emission. This can be inferred from the probability of 3-body decay as a function of dissipated energy. All this is to prove that there are honest-to-goodness $\mathrm{CN}$ in the exit charne] which decay as such, not only insofar as the common $n, p$, and alpha-particle channels are concerned, but also with respect to the more exotic complex fragment emission as well.

Coming back to gamma rays, the experiment measured them up to $E_{\gamma}=60 \mathrm{MeV}$ for 10 bins of total-kinetic-energy loss (TKEL). The ungated gamma rays look very much like those measured in other reactions, which have been interpreted in terms of nucleon-nucleon bremsstrahlung. However, when these spectra are gated with different bins of TKEL, a very different picture emerges, suggesting an exit channel rather than an entrance channel origin.

In Fig. 7 three spectra are shown covering the TKEL range of the experiment. Notice how the high excitation energy bin is associated with the stiffest gamma-ray tail while the low excitation energy bin is associated with the softest. In Fig. $8 \mathrm{a}$ this is shown more clearly by plotting the slope parameters ys TKEL. The square root-like dependence of these two quantities is very suggestive and one is tempted (and should be!) to interpret the slope parameter as a temperature. When, the 


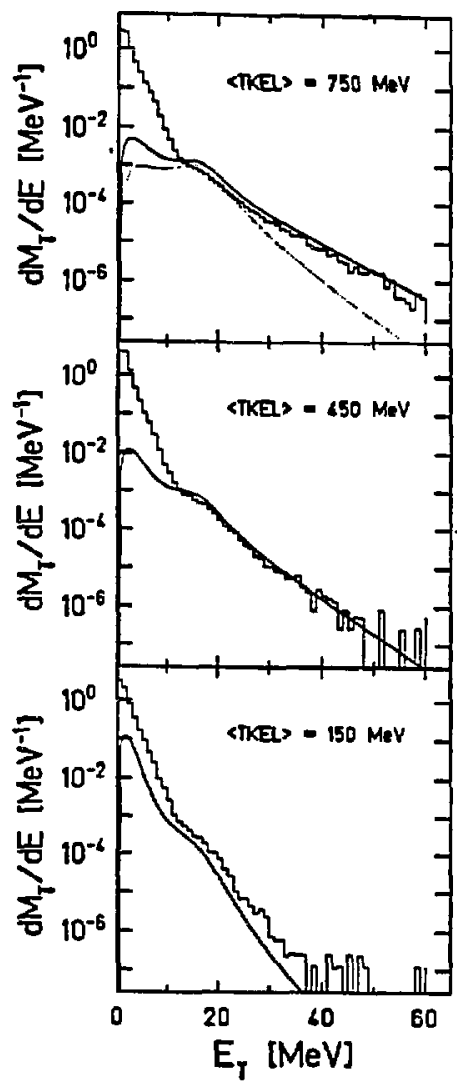

FIGURE 7

Gamma-ray spectra for three different bins in total kinetic enegy loss (TKEL). The solid curves represent statistical model calculations. The dotted curve is obtained in the same way as the solid curve except for the elimination of the quasideuteron component in the gamma-ray cross section.

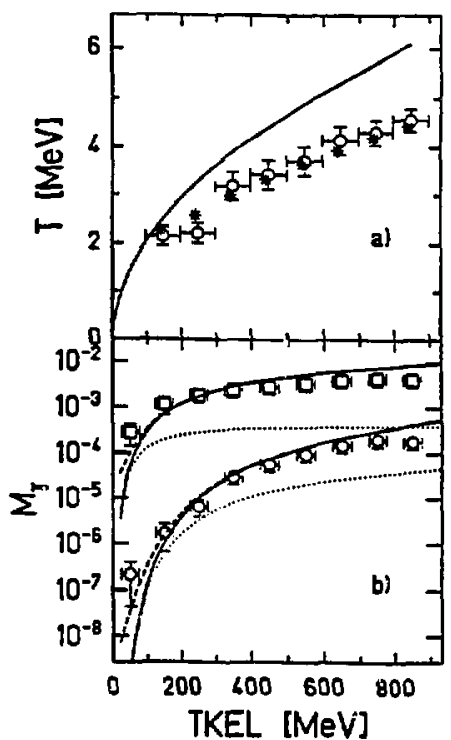

FIGURE 8

a) "Temperatures" of Boltzman fits to measured (open circles) and celculated (stars) gamma-ray spectra. The solid line denotes the primary temperature of the fragments which has been calculated from the energy loss. b) Experimental and theoretical multiplicities of hard photons with energies 215 (squares) and $\geq 30 \mathrm{MeV}$ (circles), respectively. The different lines are the result of a statistical model calculation and show the first chance contribution (dotted line), the sum over all generations (solid line) and the effect of the experimental binning of the excitation energy (dashed line).

integrated multiplicities with lower bounds of 15 and $30 \mathrm{MeV}$ are plotted versus the fragment excitation energy (sec Fig. 8b), they reveal a dependence typical of CN decay. 
This evidence does not come totally unexpected. We know that there are two $\mathrm{CN}$ in the exit channel. We also know that they decay as such by light particle emission and by complex fragment emission. Why should they not decay by gamma-ray emission? Rerhaps there are additional sources for the gamma rays, like incoherent bremsstrahlung, etc., but we know for sure that those compound nuclei must emit gamma rays. So let us calculate this emission probability. We can calculate the gamma-ray decay width in an "almest" model independent way from detailed balance and the inverse cross section:

$$
\begin{gathered}
P\left(E_{\gamma}\right)=\frac{\Gamma\left(\varepsilon_{\gamma}\right)}{\hbar}=\frac{\theta \pi}{c^{2} h^{3} \rho(E)} \sigma\left(\varepsilon_{\gamma}\right) \rho\left(E-\varepsilon_{\gamma}\right) \varepsilon_{\gamma}^{\gamma} \\
\equiv \frac{8 \pi}{c^{2} h^{3}} \sigma\left(\varepsilon_{\gamma}\right) \varepsilon_{\gamma}^{2} \theta^{-\varepsilon_{\gamma} T} .
\end{gathered}
$$

The inverse cross section is fairly well knowa experimentally. In the low energy region between 6 $20 \mathrm{MeV}$, it is dominated by the giant dipole resonance, while above this energy the quasideuteron mechanism prevails. The temperature $T$ can be calculated from the excitation energy as $E_{x}=a T^{2}$. In the actual decay, gamma-ray emission competes with $\mathrm{n}, \mathrm{p}$ and alpha-particle emissions which can be calculated in a similar fashion. In this way we can generate the "first chance" ga:nma-ray emission probability ys. excitation energy:

$$
P_{\gamma}\left(\varepsilon_{\gamma}\right)=\frac{\Gamma\left(\varepsilon_{Y}\right)}{\Gamma_{T}} \equiv \frac{\Gamma\left(\varepsilon_{\gamma}\right)}{\Gamma_{n}+\Gamma_{p}+\Gamma_{\alpha}+\ldots . .} .
$$

At this point one proceeds trivially to calculate the $2^{\text {nd }}, 3^{\text {nd }}$ etc. chance emission probability. The overall sum can be compared with experiment. In Fig. 7 we see that this calculation reproduces almost perfectly the gamma-ray energy spectra from is MeV up to $60 \mathrm{MeV}$ for all the TKEL bins, both qualitatively and quantitatively. The slope parameters of the calculated spectra can also be compared with the data. This is shown in Fig. $8 \mathrm{a}$ and again the fit is essentially farfect. The solid line in the figure represents the initial calculated temperature. The actual slope parameter is somewhat smaller due to the substantial presence of higher chance emission at the highest energies. Similarly the integrated gamma-ray multiplicities are equally well reproduced by the calculation, (see Fig. 8b). We are left with the inescapable conciusion that all of the gamma rays observed experimentally actually come from the statustical emission of the fragments. No room is left here for any other mechanism!

Somebody might object by saying, and perhaps by showing, that "other" theories fit the data almust as well and that there is no reason to choose one "theory" over aisother. The point is that our calculation is really no theory to speak about. We know that there are two $\mathrm{CN}$ in the exit channel, emitting light particles and complex fragments, because their decay products have been measured and their statistical properties verified. Therefore, we kiow that these CN must aiso emit gamma rays. All we have done is to calculate, as it were, the "background" gamma rays coming from $\mathrm{CN}$ 
decay. Any other "theory" should be tested only after this "background" has been subtracted. In this case nothing is left and the matrer is settled.

\section{RION EMISSION AND ENERGY FLUCTUATIONS}

It would be interesting to check how much of the $\pi^{\circ}, \pi^{ \pm}$production in intermediate-energy heavy-ion reactions can be explained in terms of emission from the $\mathrm{CN}$ present in the exit channel. Unfortunately, this will have to wait for more complete experiments, although it is an easy guess that, in certain low energy reactions, the $\mathrm{CN}$ contribution may not be negligible and must be evaluated.

In the case of gamma-ray emission discussed above, two hot fragments are assumed to be present in the exit channel. The calculation was performed by assigning to each fragment one-half of the available et.ergy. This may be correct on the average, but fluctuations may be present, thermal or otherwise, that may have surprising effects. In general, the role of the fluctuations in the energy distribution between two or more fragments becomes more important as the barrier or negative $Q$ value for the decay under consideration becomes bigger. Such would be the case in pion emission where the emitter must invest an energy at least equal to the pion mass in order to emit it. Let us consider the case of two nuclei in the exit cliannel with mass $A_{1}=A_{2}=A$ and with average excitation energy $E_{1}=E_{2}=E$. The probability of emitting a pion is given by:

$$
\frac{\Gamma_{g}}{\Gamma_{n}+\Gamma_{p}+\Gamma_{a}+\ldots}
$$

which is controlled by the ratio of the width $\Gamma_{\boldsymbol{x}}$ 'w that of the most probable channel like $\Gamma_{\mathbf{n}}$.

The integrated neutron decay width can be written as:

$$
\Gamma_{n}=\frac{2}{2 \pi \varphi(E)} \frac{8 \pi m_{n}}{n^{2}} \sigma_{n} T_{n}^{2} \rho\left(E-B_{n}\right)
$$

where $m_{n}$ and $B_{n}$ are the neution mass and binding energy respectively, $\sigma_{n}$ is the inverse cross section; $\rho(E), \rho\left(E-b_{n}\right)$ are the level densities of the $C N$ and of the residual nucleus, respectively, and $T_{n}$ is the temperature of the residual nucleus at excitation energy $E-B_{n}$.

The differential pion decay width is:-

$$
\Gamma_{\pi}^{*}(\varepsilon)=\frac{1}{2 \pi \rho(E)} \frac{8 \pi m_{\pi} \sigma_{\pi}}{h^{2}} \varepsilon \rho\left(E-m_{\pi}-\varepsilon\right)
$$

where $m_{\pi}, \varepsilon$ are the pion mass and kinetic energy, respectively, $\sigma_{\pi}$ the inverse cross section and the other quantities are the same as in the previous equation. 
For the ratio we have:

$$
\frac{\Gamma_{\pi}(\varepsilon)}{\Gamma_{n}}=\frac{1}{2} \frac{m_{x}}{m_{n}} \frac{\sigma_{n}}{\sigma_{n}} \frac{1}{T_{n}^{2}} \frac{\varepsilon \rho\left(E-m_{x}-\varepsilon\right)}{\rho\left(E-B_{n}\right)} .
$$

Now let us suppose that a fluctuation in the energy partition cecurs such that fragment 1 has energy $E+x$ and fragment 2 has energy $E-x$. The emission probability per fragment becomes:

$$
\frac{\Gamma_{n}(\varepsilon, x)}{\Gamma_{n}}=\frac{1}{2} \frac{\Gamma_{n}(\varepsilon)}{\Gamma_{n}}\left[\frac{\exp \times T_{\pi}}{\exp \times T_{n}}+\frac{\exp -x / T_{n}}{\exp \times / T_{n}}\right]=\frac{\Gamma_{x}(\varepsilon)}{\Gamma_{n}} \cosh \times T_{\text {aff }} \text {. }
$$

where $T_{\pi}$ is the ternperature calculated for an energy $E-m_{\pi}-\varepsilon$ and

$$
T_{\text {eff }}^{-1}=T_{k}^{-1}-T_{n}^{-1} \text {. }
$$

If the fluctuations are distributed as:

$$
P(x)=\frac{1}{\sqrt{2 \pi \sigma^{2}}} \exp -x^{2} / 2 \sigma^{2},
$$

the average emission probability becomes:

$$
\begin{gathered}
\frac{\overline{\Gamma_{n}(\varepsilon)}}{\Gamma_{n}}=\frac{\Gamma_{x}(\varepsilon)}{\Gamma_{H}} \frac{1}{\sqrt{2 \pi 0^{2}}} \int \exp -x^{2} / 2 \sigma^{2} \cosh x T_{\text {eff }} d x \\
=\frac{\Gamma_{\pi}(\varepsilon)}{\Gamma_{n}} \exp \frac{\sigma^{2}}{2 T_{\text {of }}^{2}}=\frac{\Gamma_{x}(\varepsilon)}{\Gamma_{n}} F\left(\sigma, T_{\text {.ff }}\right)
\end{gathered}
$$

For thermal fluctuations the variance is:

$$
\sigma^{2}=2 T^{3} \frac{a_{1} a_{2}}{a_{1}+a_{2}},
$$

where $T$ is the common temperature of the fragments before any emission has occurred, and $a_{1}, a_{2}$ are the level density parameters of the fragments. For a symmetric system

$$
\sigma^{2}=a T^{3}
$$

and

$$
F=\exp \frac{a T^{3}}{2 T^{2}}
$$

In Fig. 9, we have plotted the thermal enhancement factor in pion emission as a function of the toral excitation energy of the fragments. The enhancement, of course, rapidly increases with decreasing excitation energy. Simiiarly in Fig. 10 we have plotted the expected pion spectra if two fragments of mass $A=100$ each share a toul of $800 \mathrm{MeV}$ excitation energy. In one case we have.assumed an 


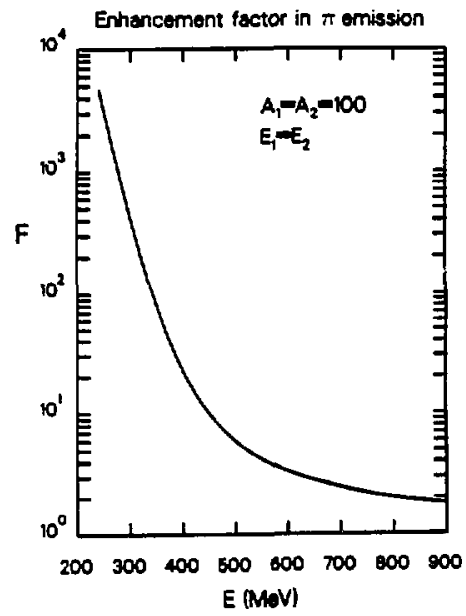

FIGURE 9

Thermal fluctuations enhancement factor in the emission of pions as a function of the total excitation energy.

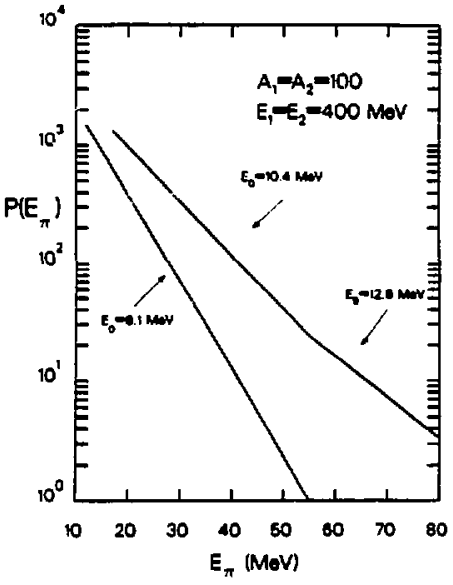

FIGURE 10

Pion spectra calculated with and without the enhancement factor due to thermal fluctuations.

exact partition of the energy and in the other we have allowed for thermal energy fluctuations. The two slope parameters are quile different, the fluctuations allowing for a substantially larger spectral temperature.

The rather spectacular increase in spectral temperature appears less spectacular when one considers that its origin lies mainly in the error introduced by the sharp energy partition. Should one consider the combined system on one hand and the two fragments in contact on the other, one has the obvious equality:

$$
\rho_{c .3 .}(E)=\int \rho_{1}(x) \rho_{2}(E-x) d x,
$$

where $p_{\text {c.s. }}$ (E) is the level density of the two framents considered as one combined system.

In other words the folding of the level densities of the two fragments calculated over the whole range of energy fluctuations is equal to the level density of the combined system at the fixed total energy $E$. This means that it does not matter if one has one, two, or more fragments in thermal equilibrium. Therefore the spectral temperature of the pions emitted by the two fragments cannot exceed the spectral temperanure that would arise if the pions were emitted by the combined system. This is true only if the energy fluctuations are thermal. If the fluctuations are dynamical in nature and larger than the corresponding thermal nuctuations, then the spectral temperature can indeed be larger than the upper limit described above. 
These general consideration cast some doubis on thermal models that rely on exit channel clusterization to achieve high pion emission probabilities.

\section{CONCLUSIONS}

From this brief discussion, one can conclude that compound nuclei, which dominate reactions at low energies, still play a big role at intermediate energies. The increase in excitation energy enhances processes that were very improbable at low energies, like the emission of complex fragments, high energy gamma rays and even pions. Also, the larger excitation energy available permits extensive sequential emission of complex fragments, thus simulating true multifragment exit channels. Finally, energy fluctuations among fragments can lead to enhancements in the emission probabilities and in the apparent spectral temperatures of very improbable channels.

\section{REFERENCES}

I) J. Aichelin and J. Hüfner, Phys. Lett. 136B (1984) 15.

2) J.E. Finn et al., Phys. Rev. Lett. 49 (1982) 1321.

3) D.H.E. Gross et al, Z. Phys. A309 (1982) 41.

4) J.P. Bondorf et al., Nucl. Phys. A.443 (1985) 321.

5) K.D. Hildenbrand et al., Proc. Int. Workshop on Gross Properties of Nuclei and Nuclear Reactions XIII, Hirschegg, Austria, edited by f. Feldmeier (1985) 111.

6) A. Olmi et al., Europhys. Lett. 4 (1987) 1121. A. Olmi, Proceedings of the 8th High Energy Heavy Ion Study (1988) 288, Lawrence Berkeley Laboratory, LBL-24580.

7) iM. Morjean et al., Phys. Lett. 203B (1988) 215.

8) L.G. Moretto and G.J. Wozniak, Prog. in Part. and Nucl. Phys. (1988) 'in prini', LBL24558, and references therein.

9) L.G. Sobotka et al., Phys. Rev. Lett. 51 (1983) 2187.

M.A. MeMahan et al., Phys. Rev. Lett. 54 (1985) 1995.

10) L.G. Moretto, Nucl. Phys. A247 (1975) 211.

11) R.J. Charity et al., Phys. Rev. Lett. 56 (1986) 1354.

R.J. Charity et al., Nucl. Phys. A.476 (1988) 516.

R.J. Charicy et al., Nucl. Phys. A "in print" (1988), LBL-22448.

D.R. Bowman et al., Phys. Lett. B 189 (1987) 282.

12) W. Cassing et al., Phys. Lett. 181B (1986) 217.

H. Nifenecker et al., Nucl. Phys. A442 (1985) 478.

13) N. Hermann et al., Phys. Rev. Lett. 60 (1988) 1630. 


\section{DISCLAIMER}

This report was prepared as an account of work sponsored by an agency of the United Slates Goyernment. Neither the United States Government nor any agency thereof, nor any of their employees, nuakes any warranty, express or implied, or assumes any legal liability or responibility for the accurncy, completeness, or usefulness of any infomation, apparatus, product, or process disclosed, or represents that its use would not infringe privately owned rights. Reference herein to eny specific commercial product, process, or service by trade name, trademart, manufacturer, or otherwise does not nocessarily constitute or imply its endorsement, recommendation, or favoring by the United States Government or any agency thereof. The views and opinions of authors expressed herein do not necessarily state of reflect those of the United States Government or any agency thereof. 